Supporting Information

\title{
Origin of the High Donor-Acceptor Composition Tolerance in Device Performance and Mechanical Robustness of All-Polymer
} Solar Cells

Jin-Woo Lee,,$^{\dagger, \perp}$ Boo Soo Ma, $,^{\ddagger}, \perp$ Joonhyeong Choi, ${ }^{\dagger}$ Junbok Lee, ${ }^{\dagger}$ Seungjin Lee, ${ }^{\dagger}$ Kin Liao, $^{\S}$ Wonho Lee, ${ }^{*}, \|$ Taek-Soo Kim, ${ }^{*, \ddagger}$ and Bumjoon J. Kim ${ }^{*},+$

${ }^{\dagger}$ Department of Chemical and Biomolecular Engineering, ${ }^{\ddagger}$ Department of Mechanical Engineering, Korea Advanced Institute of Science and Technology (KAIST), Daejeon, 34141, Republic of Korea

${ }^{\S}$ Department of Mechanical Engineering, Khalifa University, Abu Dhabi 127788, United Arab Emirates

" Department of Polymer Science and Engineering, Kumoh National Institute of Technology, Gumi-si, Gyeongbuk 39177, Republic of Korea

*(B. J. Kim) E-mail: bumjoonkim@kaist.ac.kr

*(T.-S. Kim) E-mail: tskim1@kaist.ac.kr

*(W. Lee) E-mail: 1holee@kumoh.ac.kr 


\section{Table of Contents}

\section{Supplementary Figures}

Figure S1. SEC curves of P(NDI2OD-T2) polymers with different $M_{\mathrm{n}}$ values.

Figure S2. DSC thermograms of $\mathrm{P}_{\mathrm{H}}, \mathrm{P}_{\mathrm{M}}$, and $\mathrm{P}_{\mathrm{L}}$ for (a) second heating and (b) second cooling cycles. (c) Normalized solution absorption spectra of $\mathrm{P}_{\mathrm{H}}, \mathrm{P}_{\mathrm{M}}$, and $\mathrm{P}_{\mathrm{L}}\left(0.01 \mathrm{mg} \mathrm{mL}^{-1}\right.$ in CB). (d) Film absorption coefficients of PBDB-T, $\mathrm{P}_{\mathrm{H}}, \mathrm{P}_{\mathrm{M}}$, and $\mathrm{P}_{\mathrm{L}}$.

Figure S3. Normalized (a) $V_{\text {oc }}$ and (b) FF plots of PBDB-T:acceptors at different D:A ratios. Figure S4. EQE spectra of (a) PBDB-T:P $\mathrm{P}_{\mathrm{M}}$, (b) PBDB-T:ITIC, and (c) PBDB-T:PC ${ }_{71} \mathrm{BM}$ at different D:A Ratios.

Figure S5. $V_{\text {eff }}$ Vs. $J_{\text {ph }}$ curves for (a) PBDB-T:P $\mathrm{P}_{\mathrm{L}}$ (b)PBDB-T: $\mathrm{P}_{\mathrm{M}}$, and (c)PBDB-T: $\mathrm{P}_{\mathrm{H}}$. Figure S6. 2D-GIXS of the pristine donor and acceptor polymers in thin films.

Figure S7. 2D-GIXS of the PBDB-T: $P_{A}$ blend films as functions of $P_{A} M W$ and D:A ratios.

Figure S8. GIXS linecut profiles of (a) PBDB-T: $\mathrm{P}_{\mathrm{L}}$, (b) PBDB-T: $\mathrm{P}_{\mathrm{M}}$, and (c) PBDB-T: $\mathrm{P}_{\mathrm{H}}$ blend films in the out-of-plane direction.

Figure S9. (a) Schematics of double cantilever beam (DCB) test setup, (b) load-displacement curve, and (c) structure of the specimen after the DCB test.

Figure S10. AFM height images of BHJ film surfaces before DCB test in (a) PBDB-T: $\mathrm{P}_{\mathrm{L}}$, (b) PBDB-T:P $P_{M}$ and (c) PBDB-T:PH blends at different D:A ratios. Scale bars are $1 \mu \mathrm{m}$.

Figure S11. (a) Schematics of the pseudo free-standing tensile test setup. (b) Dog-bone shaped BHJ film floated on the water surface, (c) BHJ film fixed to the PDMS-coated aluminum grips, and (d) fractured BHJ film after the tensile test. (e) Stress-strain curves with D:A ratio of 1:1.

\section{Supplementary Tables}

Table S1. D:A ratio dependent photovoltaic performances and film thickness of PBDBT:acceptors depending on the acceptor material types and $P_{\mathrm{A}} \mathrm{MW}$.

Table S2. Mobilities from SCLC method and $P(E, T)$ for various blend ratios of PBDBT:acceptors.

Table S3. Summary of $d$-spacing of the scattering peaks from PBDB-T and P(NDI2OD-T2) pristine films.

Table S4. Summary of $L_{\mathrm{c}}$ and normalized $L_{\mathrm{c}}$ of (001) peaks in the in-plane direction for PBDB$\mathrm{T}: P_{\mathrm{A}} \mathrm{S}$ in different $\mathrm{D}$ :A ratios.

Table S5. Elastic modulus, crack onset strain (COS), toughness and cohesion energy $\left(G_{c}\right)$ for PBDB-T: $P_{\mathrm{AS}}$ in different $\mathrm{D}:$ A ratios. 


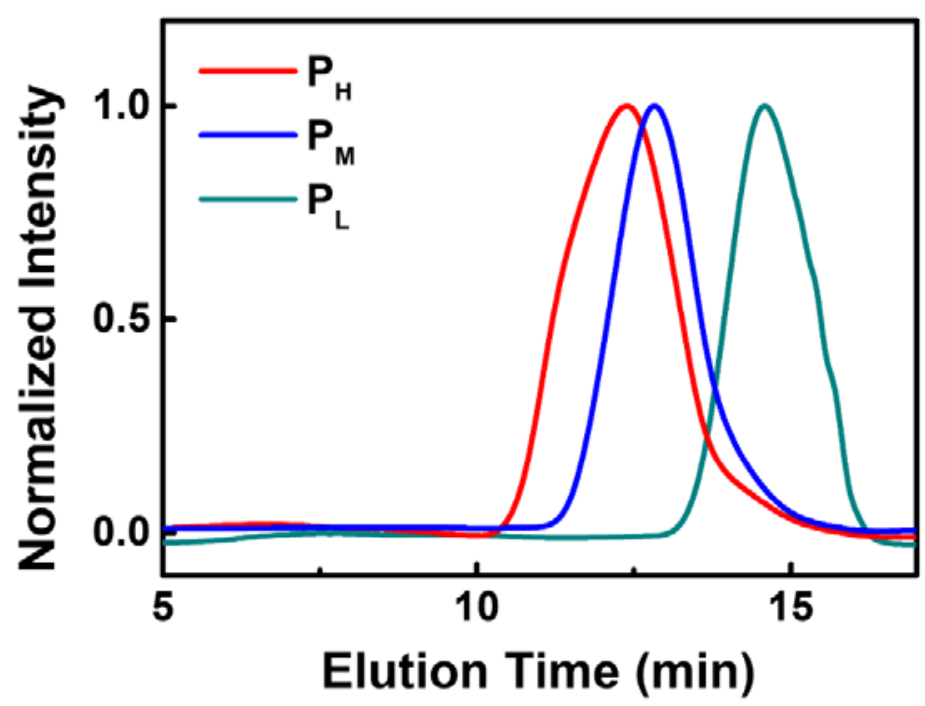

Figure S1. SEC curves of P(NDI2OD-T2) polymers with different $M_{\mathrm{n}}$ values.

(a)

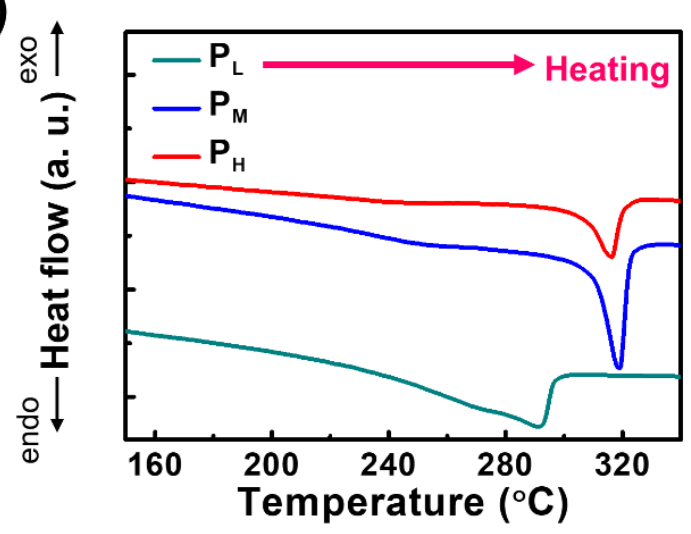

(c)

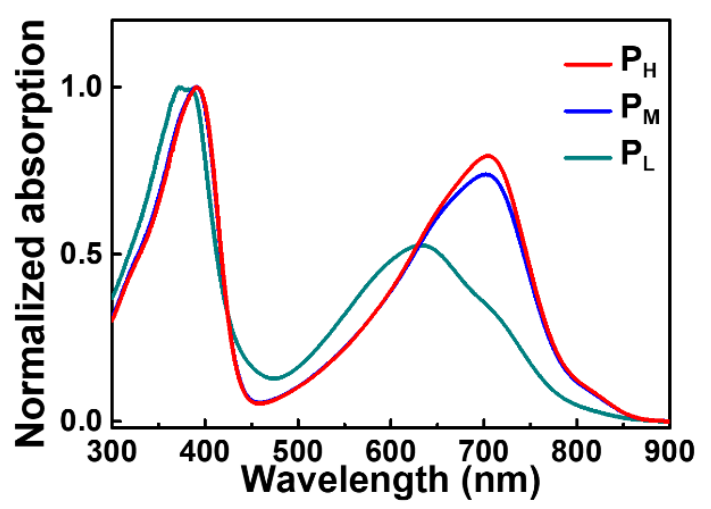

(b)

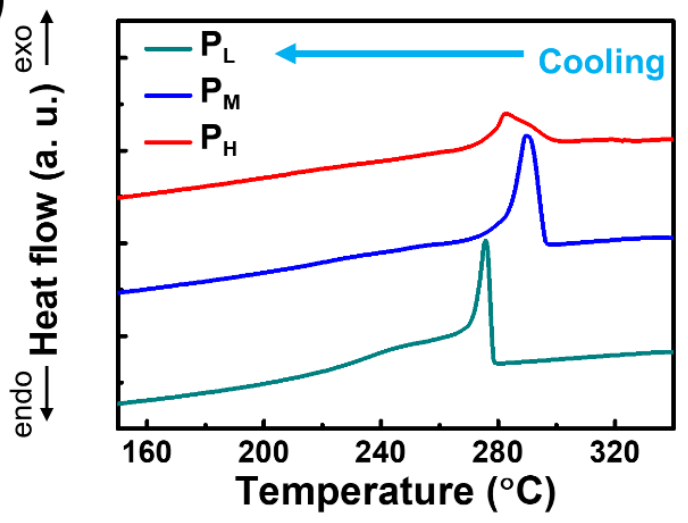

(d)

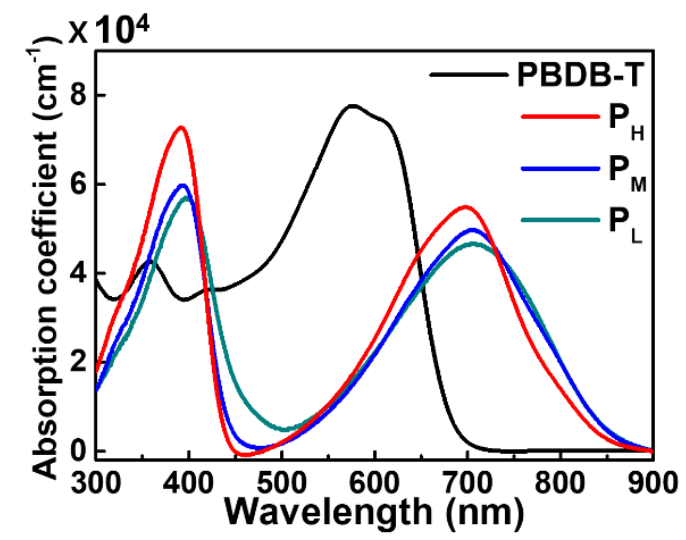

Figure S2. DSC thermograms of $\mathrm{P}_{\mathrm{H}}, \mathrm{P}_{\mathrm{M}}$, and $\mathrm{P}_{\mathrm{L}}$ for (a) second heating and (b) second cooling cycles. (c) Normalized solution absorption spectra of $\mathrm{P}_{\mathrm{H}}, \mathrm{P}_{\mathrm{M}}$, and $\mathrm{P}_{\mathrm{L}}\left(0.01 \mathrm{mg} \mathrm{mL}^{-1}\right.$ in CB). (d) Film absorption coefficients of PBDB-T, $\mathrm{P}_{\mathrm{H}}, \mathrm{P}_{\mathrm{M}}$, and $\mathrm{P}_{\mathrm{L}}$. 
Table S1. D:A ratio dependent photovoltaic performances and film thickness of PBDB$\mathrm{T}$ :acceptors depending on the acceptor material types and $P_{\mathrm{A}} \mathrm{MW}$.

\begin{tabular}{|c|c|c|c|c|c|c|}
\hline Acceptor & $\begin{array}{l}\mathrm{D}: \mathrm{A} \\
\text { ratio }\end{array}$ & $\begin{array}{l}V_{\text {oc }} \\
(V)^{\mathrm{a}}\end{array}$ & $\begin{array}{c}J_{\text {sc }} \\
\left(\mathrm{mA} \mathrm{cm}^{-2}\right)^{\mathrm{a}} \\
\end{array}$ & $\mathbf{F F}^{\mathbf{a}}$ & $\begin{array}{c}\text { PCE }_{\text {avg }}^{\text {a }}\left(P^{\prime} C E_{\max }\right) \\
(\%)\end{array}$ & $\begin{array}{c}\text { Thickness }^{\mathrm{b}} \\
\text { (nm) }\end{array}$ \\
\hline \multirow{6}{*}{$\mathbf{P}_{\mathbf{L}}$} & $1: 3$ & $0.85 \pm 0.01$ & $3.63 \pm 0.09$ & $0.52 \pm 0.04$ & $1.60 \pm 0.05(1.74)$ & 87 \\
\hline & $1: 1$ & $0.89 \pm 0.01$ & $9.61 \pm 0.14$ & $0.60 \pm 0.02$ & $5.13 \pm 0.18(5.30)$ & 91 \\
\hline & $3: 1$ & $0.88 \pm 0.00$ & $6.38 \pm 0.38$ & $0.45 \pm 0.02$ & $2.53 \pm 0.25(2.78)$ & 97 \\
\hline & $5: 1$ & $0.89 \pm 0.00$ & $3.06 \pm 0.11$ & $0.42 \pm 0.03$ & $1.14 \pm 0.05$ & 101 \\
\hline & 10:1 & $0.90 \pm 0.01$ & $1.21 \pm 0.04$ & $0.35 \pm 0.02$ & $0.38 \pm 0.04(0.55)$ & 110 \\
\hline & 15:1 & $0.90 \pm 0.00$ & $0.89 \pm 0.07$ & $0.31 \pm 0.02$ & $0.25 \pm 0.06(0.38)$ & 114 \\
\hline \multirow{6}{*}{$\mathbf{P}_{\mathbf{M}}$} & $1: 3$ & $0.86 \pm 0.00$ & $9.58 \pm 0.23$ & $0.51 \pm 0.01$ & $4.20 \pm 0.13(4.40)$ & 104 \\
\hline & $1: 1$ & $0.89 \pm 0.01$ & $13.09 \pm 0.21$ & $0.56 \pm 0.01$ & $6.52 \pm 0.07(6.68)$ & 105 \\
\hline & $3: 1$ & $0.89 \pm 0.01$ & $10.22 \pm 0.19$ & $0.52 \pm 0.03$ & $4.73 \pm 0.13(4.80)$ & 108 \\
\hline & $5: 1$ & $0.88 \pm 0.00$ & $9.58 \pm 0.10$ & $0.48 \pm 0.01$ & $4.05 \pm 0.09(4.21)$ & 105 \\
\hline & 10:1 & $0.90 \pm 0.00$ & $8.30 \pm 0.20$ & $0.39 \pm 0.02$ & $2.91 \pm 0.25(3.01)$ & 103 \\
\hline & 15:1 & $0.91 \pm 0.01$ & $6.91 \pm 0.39$ & $0.33 \pm 0.02$ & $2.07 \pm 0.23(2.14)$ & 107 \\
\hline \multirow{6}{*}{$\mathbf{P}_{\mathbf{H}}$} & $1: 3$ & $0.88 \pm 0.01$ & $11.58 \pm 0.10$ & $0.50 \pm 0.03$ & $5.10 \pm 0.11(5.30)$ & 109 \\
\hline & $1: 1$ & $0.89 \pm 0.00$ & $13.34 \pm 0.23$ & $0.58 \pm 0.02$ & $6.89 \pm 0.16(7.02)$ & 101 \\
\hline & $3: 1$ & $0.89 \pm 0.01$ & $13.15 \pm 0.26$ & $0.55 \pm 0.02$ & $6.44 \pm 0.23(6.57)$ & 104 \\
\hline & $5: 1$ & $0.90 \pm 0.00$ & $12.53 \pm 0.09$ & $0.54 \pm 0.01$ & $6.09 \pm 0.06(6.20)$ & 96 \\
\hline & 10:1 & $0.92 \pm 0.00$ & $11.47 \pm 0.11$ & $0.51 \pm 0.01$ & $5.38 \pm 0.11(5.53)$ & 108 \\
\hline & 15:1 & $0.91 \pm 0.01$ & $10.73 \pm 0.16$ & $0.46 \pm 0.02$ & $4.49 \pm 0.14(4.66)$ & 110 \\
\hline \multirow{5}{*}{ ITIC } & $1: 1$ & $0.88 \pm 0.01$ & $17.20 \pm 0.53$ & $0.56 \pm 0.01$ & $8.48 \pm 0.15(8.61)$ & 90 \\
\hline & $3: 1$ & $0.91 \pm 0.00$ & $11.91 \pm 0.11$ & $0.43 \pm 0.02$ & $4.66 \pm 0.13(4.78)$ & 101 \\
\hline & $5: 1$ & $0.88 \pm 0.00$ & $2.77 \pm 0.03$ & $0.42 \pm 0.01$ & $1.02 \pm 0.02(1.05)$ & 97 \\
\hline & $10: 1$ & $0.89 \pm 0.01$ & $1.08 \pm 0.02$ & $0.40 \pm 0.01$ & $0.38 \pm 0.02(0.41)$ & 117 \\
\hline & $15: 1$ & $0.87 \pm 0.00$ & $0.85 \pm 0.02$ & $0.38 \pm 0.01$ & $0.27 \pm 0.03(0.33)$ & 119 \\
\hline \multirow{5}{*}{$\mathbf{P C}_{71} \mathrm{BM}$} & $1: 1$ & $0.82 \pm 0.01$ & $13.63 \pm 0.06$ & $0.67 \pm 0.01$ & $7.49 \pm 0.07$ (7.55) & 85 \\
\hline & $3: 1$ & $0.89 \pm 0.01$ & $3.52 \pm 0.13$ & $0.38 \pm 0.01$ & $1.19 \pm 0.06(1.24)$ & 93 \\
\hline & $5: 1$ & $0.90 \pm 0.01$ & $2.57 \pm 0.10$ & $0.34 \pm 0.02$ & $0.79 \pm 0.02(0.83)$ & 101 \\
\hline & 10:1 & $0.86 \pm 0.01$ & $2.06 \pm 0.07$ & $0.33 \pm 0.01$ & $0.58 \pm 0.03(0.60)$ & 115 \\
\hline & $15: 1$ & $0.93 \pm 0.01$ & $1.16 \pm 0.02$ & $0.30 \pm 0.01$ & $0.32 \pm 0.02(0.36)$ & 118 \\
\hline
\end{tabular}

${ }^{\mathrm{a}}$ Average values and standard deviations were determined from more than 10 devices.

${ }^{\mathrm{b}}$ Thicknesses were determined from the avarage value of 3 different films. 
(a)

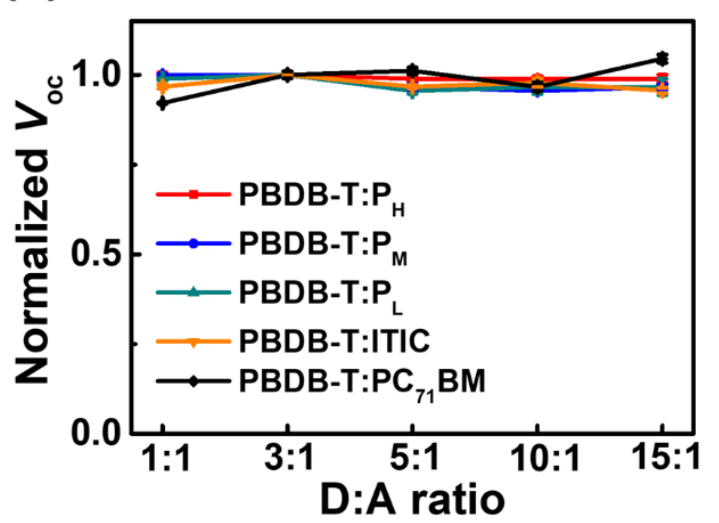

(b)

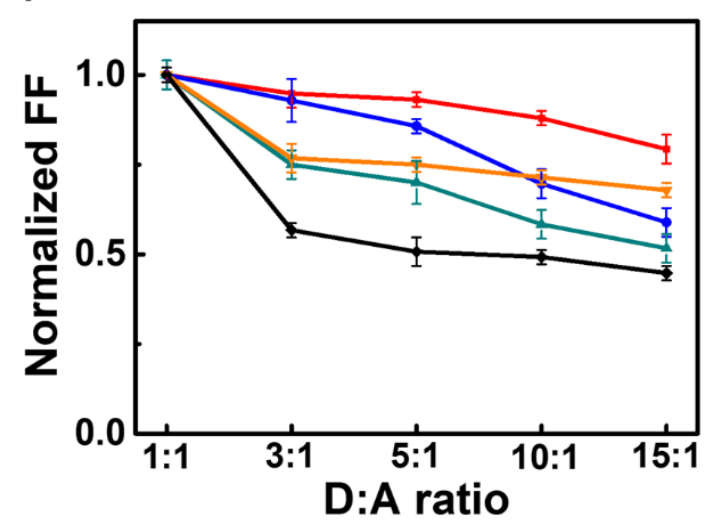

Figure S3. Normalized (a) $V_{\text {oc }}$ and (b) FF plots of PBDB-T:acceptors at different D:A ratios.

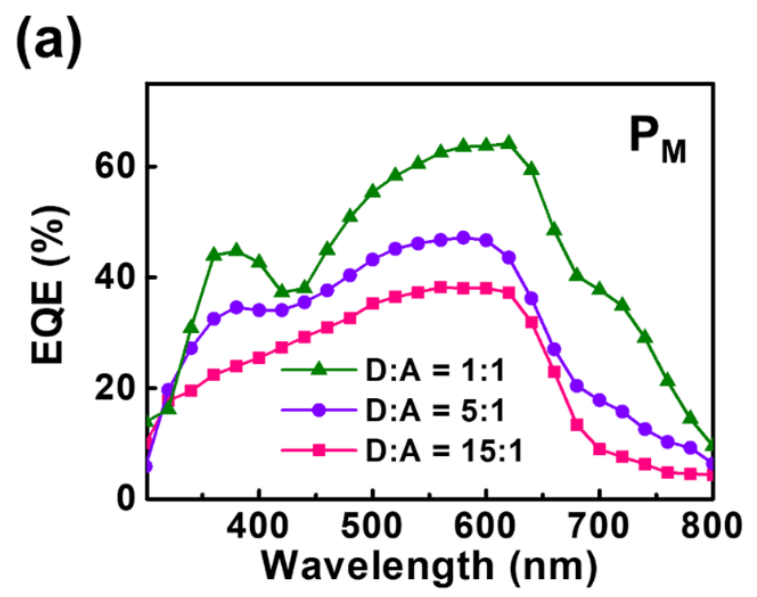

(b)

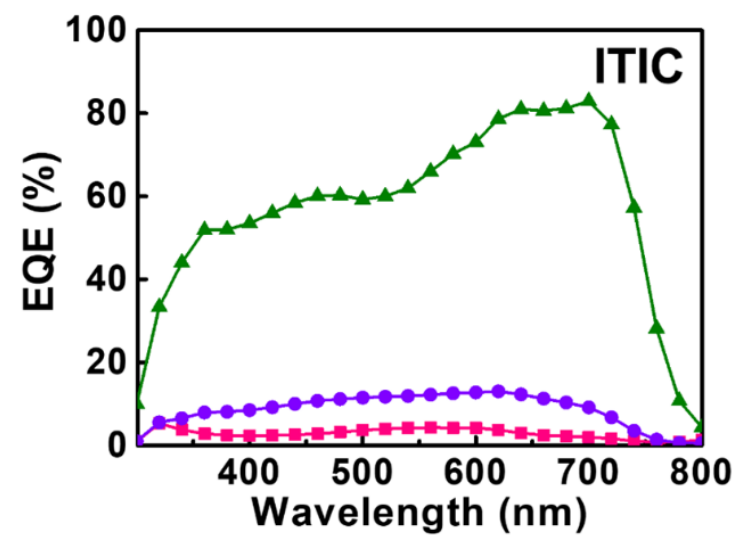

(c)

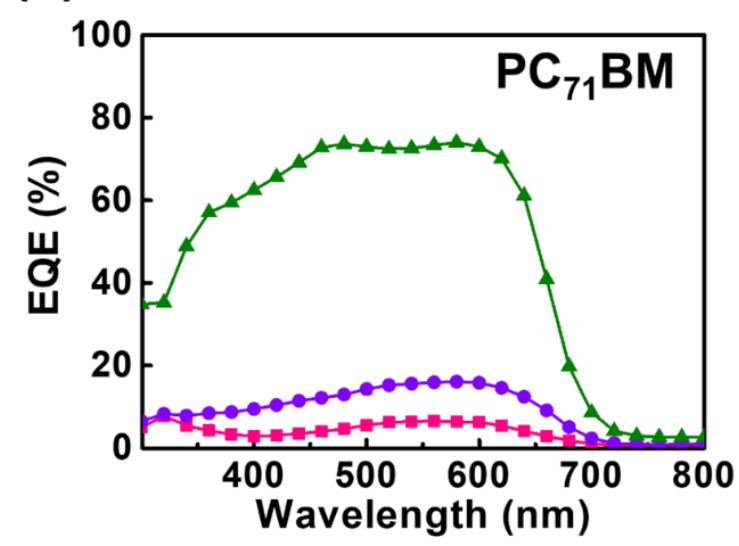

Figure S4. EQE spectra of (a) PBDB-T:PM, (b) PBDB-T:ITIC, and (c) PBDB-T:PC ${ }_{71} B M$ at different D:A ratios. 
Table S2. Mobilities from SCLC method and $P(E, T)$ for various blend ratios of PBDBT:acceptors.

\begin{tabular}{|c|c|c|c|c|c|}
\hline Acceptor & D:A ratio & $\mu_{\mathrm{e}}\left(\mathrm{cm}^{2} \mathrm{~V}^{-1} \mathrm{~s}^{-1}\right)^{\mathrm{a}}$ & $\mu_{\mathrm{h}}\left(\mathrm{cm}^{2} \mathrm{~V}^{-1} \mathrm{~s}^{-1}\right)^{\mathrm{a}}$ & $\mu \mathrm{h} / \mu \mathrm{e}$ & $P(E, T)(\%)^{b}$ \\
\hline \multirow{3}{*}{$\mathbf{P}_{\mathbf{L}}$} & $1: 1$ & $3.7 \times 10^{-5}$ & $7.4 \times 10^{-5}$ & 2.0 & 74 \\
\hline & $5: 1$ & $3.8 \times 10^{-6}$ & $1.7 \times 10^{-4}$ & 45 & 25 \\
\hline & $15: 1$ & $3.0 \times 10^{-8}$ & $3.5 \times 10^{-4}$ & $1.2 \times 10^{4}$ & 12 \\
\hline \multirow{3}{*}{$\mathbf{P}_{\mathbf{M}}$} & $1: 1$ & $4.1 \times 10^{-5}$ & $5.2 \times 10^{-5}$ & 1.3 & 81 \\
\hline & $5: 1$ & $1.2 \times 10^{-5}$ & $9.4 \times 10^{-5}$ & 7.8 & 79 \\
\hline & $15: 1$ & $4.2 \times 10^{-6}$ & $1.5 \times 10^{-4}$ & 36 & 71 \\
\hline \multirow{3}{*}{$\mathbf{P}_{\mathbf{H}}$} & $1: 1$ & $5.9 \times 10^{-5}$ & $6.6 \times 10^{-5}$ & 1.1 & 88 \\
\hline & $5: 1$ & $2.1 \times 10^{-5}$ & $6.7 \times 10^{-5}$ & 3.2 & 85 \\
\hline & $15: 1$ & $1.2 \times 10^{-5}$ & $1.1 \times 10^{-4}$ & 9.2 & 84 \\
\hline \multirow{3}{*}{ ITIC } & $1: 1$ & $1.2 \times 10^{-4}$ & $1.8 \times 10^{-4}$ & 1.5 & - \\
\hline & $5: 1$ & $5.8 \times 10^{-6}$ & $3.1 \times 10^{-4}$ & 53 & - \\
\hline & $15: 1$ & $1.4 \times 10^{-8}$ & $4.3 \times 10^{-4}$ & $3.1 \times 10^{4}$ & - \\
\hline \multirow{3}{*}{$\mathrm{PC}_{71} \mathrm{BM}$} & $1: 1$ & $1.1 \times 10^{-4}$ & $1.4 \times 10^{-4}$ & 1.3 & - \\
\hline & $5: 1$ & $2.1 \times 10^{-6}$ & $1.9 \times 10^{-4}$ & 90 & - \\
\hline & $15: 1$ & $1.8 \times 10^{-8}$ & $3.3 \times 10^{-4}$ & $1.8 \times 10^{4}$ & - \\
\hline
\end{tabular}

${ }^{a}$ The average values were obtained from at least 5 different SCLC devices. ${ }^{b} P(E, T)$ values were calculated by the equation $\left(P(E, T)=J_{\mathrm{ph}} / J_{\mathrm{ph}, \mathrm{sat}}\right)$, where $J_{\mathrm{ph}}$ is the photocurrent density at the short-circuit condition and $J_{\text {sat }}$ is the saturated photocurrent density. 
(a)

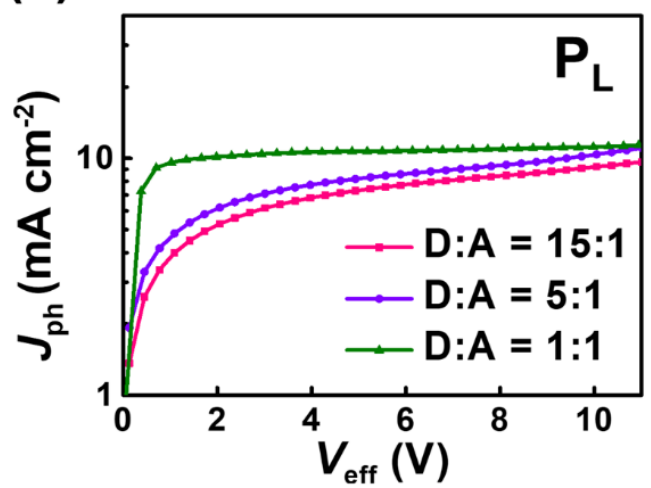

(b)

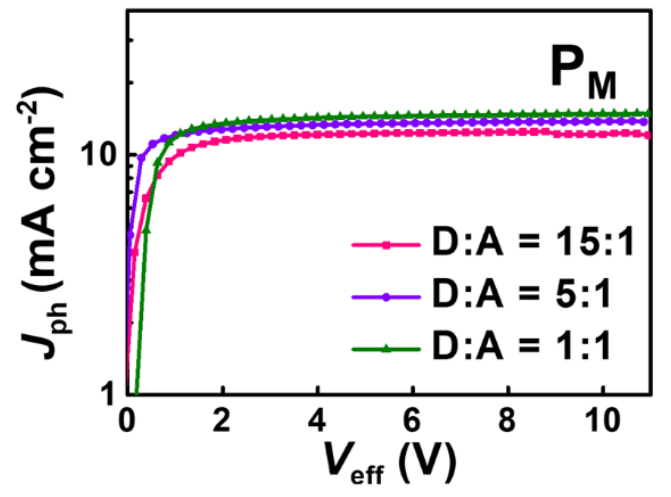

(c)

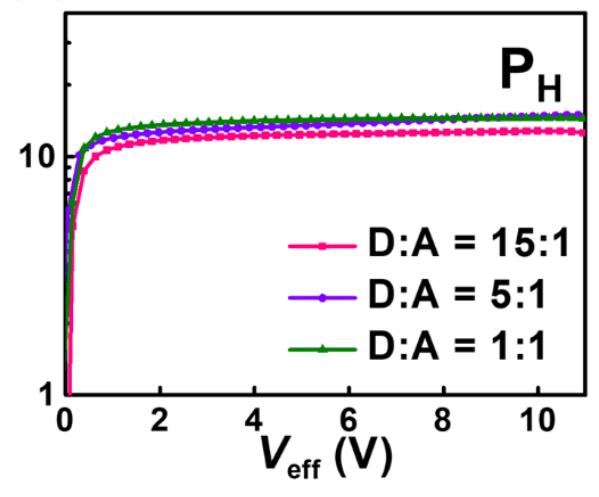

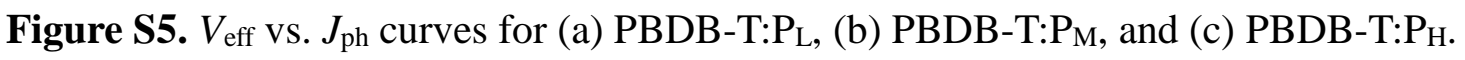



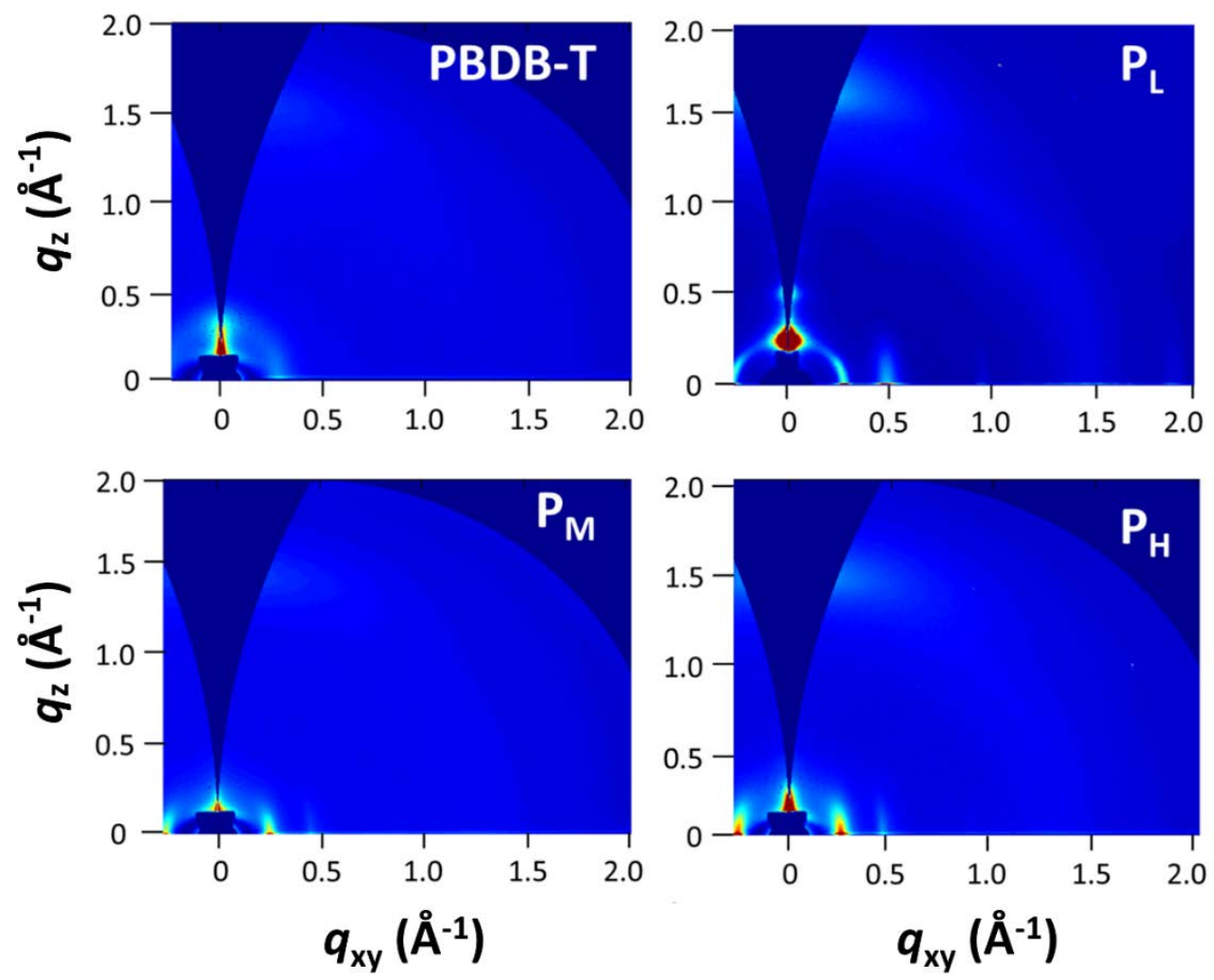

Figure S6. 2D-GIXS of the pristine donor and acceptor polymers in thin films. 

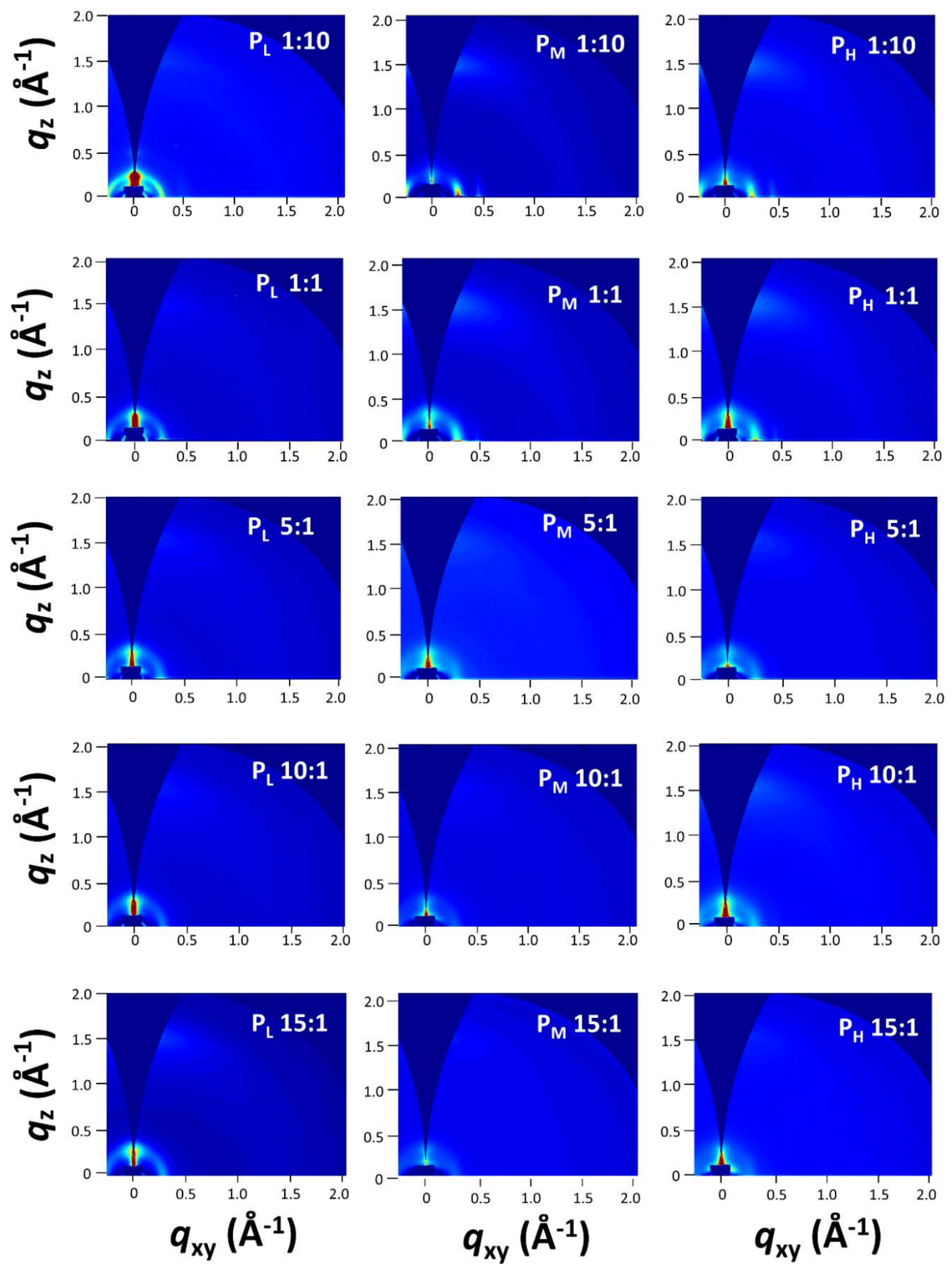

Figure S7. 2D-GIXS of the PBDB-T: $P_{\mathrm{A}}$ blend films as functions of $P_{\mathrm{A}} \mathrm{MW}$ and D:A ratios. 
(a)

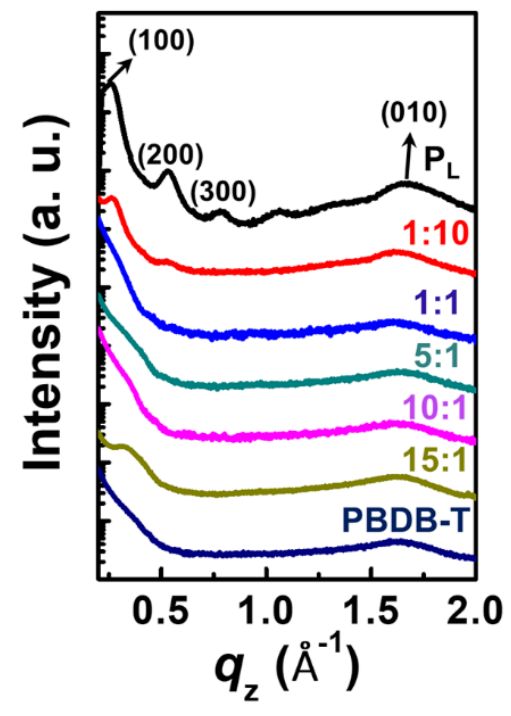

(b)

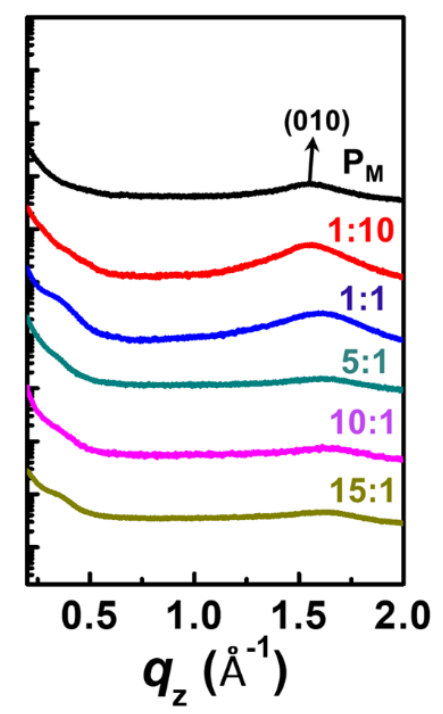

(c)

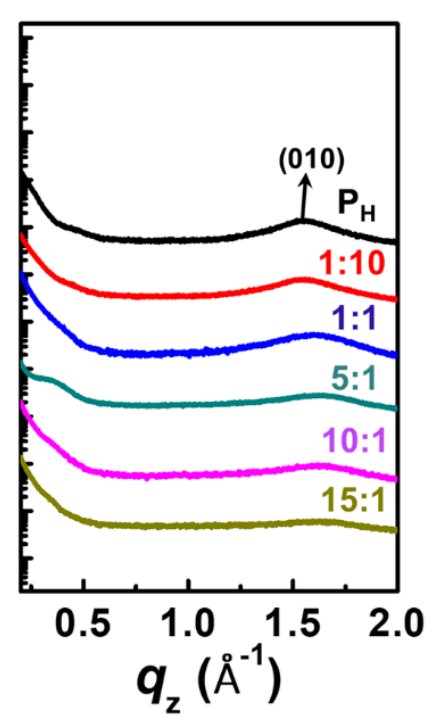

Figure S8. GIXS linecut profiles of (a) PBDB-T:P $\mathrm{P}_{\mathrm{L}}$ (b) PBDB-T:P $\mathrm{P}_{\mathrm{M}}$, and (c) PBDB-T: $\mathrm{P}_{\mathrm{H}}$ blend films in the out-of-plane direction.

Table S3. Summary of $d$-spacing of the scattering peaks from PBDB-T and P(NDI2OD-T2) pristine films.

\begin{tabular}{cccc}
\hline \multirow{2}{*}{ Polymer } & IP (100) peak & IP (001) peak & OOP (010) peak \\
\cline { 2 - 4 } & $\boldsymbol{d}$-spacing $(\AA)$ & $\boldsymbol{d}$-spacing $(\AA)$ & $\boldsymbol{d}$-spacing $(\AA)$ \\
\hline PBDB-T & 22.6 & - & 3.85 \\
$\mathbf{P}_{\mathbf{L}}$ & 24.0 & 13.8 & 3.78 \\
$\mathbf{P}_{\mathbf{M}}$ & 24.7 & 13.8 & 4.04 \\
$\mathbf{P}_{\mathbf{H}}$ & 25.2 & 13.9 & 4.06 \\
\hline
\end{tabular}


Table S4. Summary of coherence length $\left(L_{c}\right)$ and normalized $L_{c}$ of (001) peaks in the in-plane direction for PBDB-T: $P_{\mathrm{AS}}$ in different $\mathrm{D}$ :A ratios.

\begin{tabular}{cccc}
\hline \multirow{2}{*}{ Acceptor } & \multirow{2}{*}{ D:A ratio } & \multicolumn{2}{c}{ IP (001) peak } \\
\cline { 3 - 4 } & Pristine & $\boldsymbol{L}_{\mathbf{c}(\mathbf{0 0 1})}(\mathbf{n m})$ & Normalized $\boldsymbol{L}_{\mathbf{c}(\mathbf{0 0 1})}$ \\
\hline \multirow{3}{*}{$\mathbf{P}_{\mathbf{L}}$} & $1: 10$ & 5.4 & 1.00 \\
& $1: 1$ & 4.6 & 0.76 \\
& $5: 1$ & 3.1 & 0.65 \\
& $10: 1$ & - & 0.44 \\
& $15: 1$ & - & - \\
& Pristine & 8.9 & - \\
\hline \multirow{3}{*}{$\mathbf{P}_{\mathbf{M}}$} & $1: 10$ & 9.3 & 1.00 \\
& $1: 1$ & 7.0 & 1.04 \\
& $5: 1$ & 4.4 & 0.79 \\
& $10: 1$ & - & 0.49 \\
& $15: 1$ & - & - \\
\hline \multirow{3}{*}{$\mathbf{P}_{\mathbf{H}}$} & Pristine & 10.4 & 1.00 \\
& $1: 10$ & 10.2 & 0.98 \\
& $1: 1$ & 9.2 & 0.88 \\
& $5: 1$ & 6.5 & 0.63 \\
& $10: 1$ & 5.0 & 0.48 \\
& $15: 1$ & - & - \\
\hline
\end{tabular}


(a)

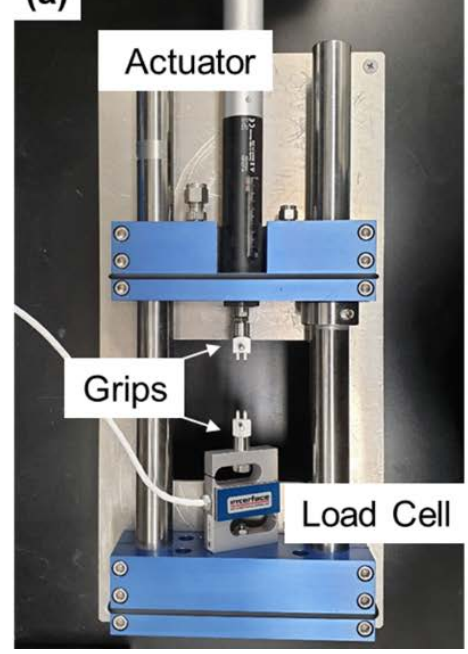

(b)

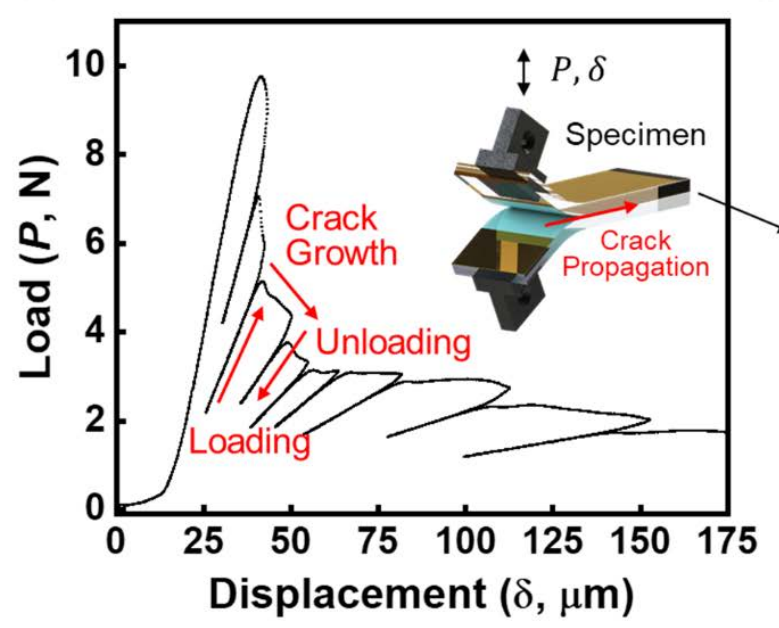

(c)

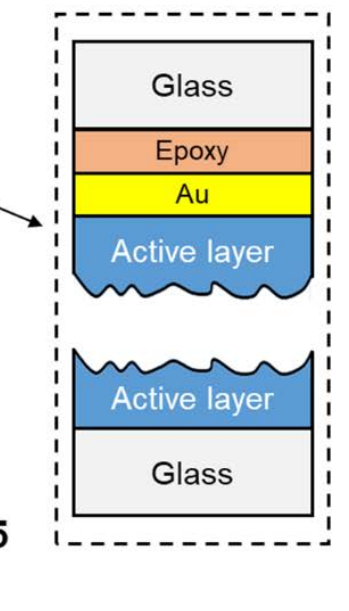

Figure S9. (a) Schematics of double cantilever beam (DCB) test setup, (b) load-displacement curve, and (c) structure of the specimen after the DCB test 
Table S5. Elastic modulus, crack onset strain (COS), toughness and cohesion energy $\left(G_{c}\right)$ for PBDB-T: $P_{\mathrm{AS}}$ in different $\mathrm{D}:$ A ratios.

\begin{tabular}{|c|c|c|c|c|c|}
\hline Acceptor & $\begin{array}{l}\mathrm{D}: \mathrm{A} \\
\text { ratio }\end{array}$ & $\begin{array}{c}\text { Elastic modulus } \\
\text { (GPa) }\end{array}$ & $\begin{array}{l}\text { COS } \\
(\%)\end{array}$ & $\begin{array}{l}\text { Toughness } \\
\left(\mathrm{MJ} \mathrm{m}^{-3}\right)\end{array}$ & $\begin{array}{c}G_{\mathbf{c}} \\
\left(\mathbf{J ~ m}^{-2}\right)\end{array}$ \\
\hline \multirow{6}{*}{$\mathbf{P}_{\mathbf{L}}$} & $1: 10$ & $0.37 \pm 0.12$ & $0.73 \pm 0.21$ & $0.01 \pm 0.01$ & $0.72 \pm 0.31$ \\
\hline & $1: 3$ & $0.42 \pm 0.02$ & $1.28 \pm 0.36$ & $0.02 \pm 0.01$ & $0.99 \pm 0.23$ \\
\hline & 1:1 & $0.65 \pm 0.12$ & $25.18 \pm 4.91$ & $2.79 \pm 0.65$ & $2.25 \pm 0.48$ \\
\hline & $5: 1$ & $1.03 \pm 0.09$ & $33.09 \pm 5.03$ & $5.96 \pm 0.54$ & $2.32 \pm 0.11$ \\
\hline & $15: 1$ & $1.30 \pm 0.05$ & $31.90 \pm 2.28$ & $8.08 \pm 0.62$ & $2.47 \pm 0.24$ \\
\hline & $1: 0$ & $1.34 \pm 0.15$ & $30.63 \pm 4.36$ & $8.73 \pm 1.48$ & $3.20 \pm 0.24$ \\
\hline \multirow{6}{*}{$\mathbf{P}_{\mathbf{M}}$} & $1: 10$ & $0.52 \pm 0.10$ & $8.50 \pm 3.70$ & $0.79 \pm 0.51$ & $2.01 \pm 0.09$ \\
\hline & $1: 3$ & $0.94 \pm 0.02$ & $23.91 \pm 0.35$ & $4.46 \pm 0.25$ & $2.13 \pm 0.14$ \\
\hline & $1: 1$ & $0.91 \pm 0.07$ & $27.94 \pm 1.84$ & $5.27 \pm 0.39$ & $2.49 \pm 0.12$ \\
\hline & $5: 1$ & $1.25 \pm 0.13$ & $32.61 \pm 1.86$ & $8.94 \pm 0.86$ & $2.53 \pm 0.16$ \\
\hline & $15: 1$ & $1.09 \pm 0.04$ & $32.75 \pm 3.95$ & $8.09 \pm 1.18$ & $2.65 \pm 0.48$ \\
\hline & $1: 0$ & $1.34 \pm 0.15$ & $30.63 \pm 4.36$ & $8.73 \pm 1.48$ & $3.20 \pm 0.24$ \\
\hline \multirow{6}{*}{$\mathbf{P}_{\mathbf{H}}$} & $1: 10$ & $0.86 \pm 0.13$ & $37.01 \pm 1.49$ & $8.18 \pm 0.69$ & $4.39 \pm 0.39$ \\
\hline & $1: 3$ & $1.07 \pm 0.06$ & $34.58 \pm 0.37$ & $9.97 \pm 0.17$ & $4.22 \pm 0.39$ \\
\hline & $1: 1$ & $0.90 \pm 0.20$ & $38.89 \pm 0.30$ & $10.05 \pm 0.32$ & $3.79 \pm 0.36$ \\
\hline & $5: 1$ & $0.93 \pm 0.12$ & $33.35 \pm 3.50$ & $7.62 \pm 0.83$ & $3.31 \pm 0.42$ \\
\hline & 15:1 & $1.04 \pm 0.15$ & $36.03 \pm 1.18$ & $9.34 \pm 0.83$ & $3.37 \pm 0.38$ \\
\hline & 1:0 & $1.34 \pm 0.15$ & $30.63 \pm 4.36$ & $8.73 \pm 1.48$ & $3.20 \pm 0.24$ \\
\hline
\end{tabular}


(a) PBDB-T:PL

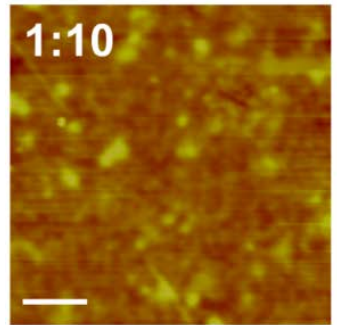

$R_{q}=2.1 \mathrm{~nm}$

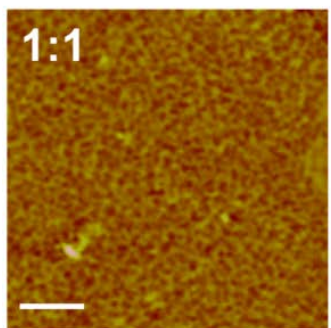

$R_{q}=2.0 \mathrm{~nm}$

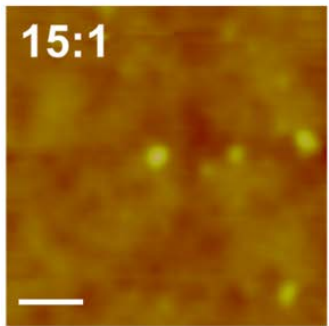

$R_{q}=1.3 \mathrm{~nm}$ (b) PBDB-T:P $P_{M}$

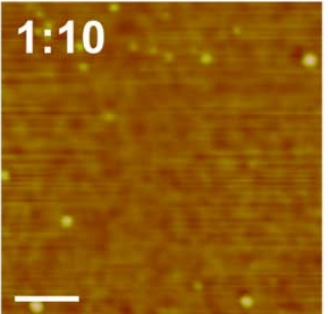

$R_{q}=1.3 \mathrm{~nm}$

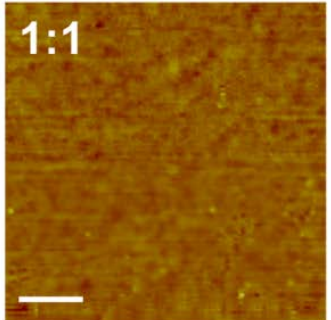

$R_{q}=1.3 \mathrm{~nm}$

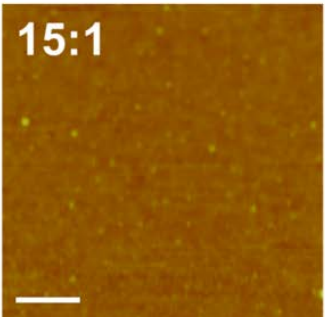

$R_{q}=0.7 \mathrm{~nm}$ (c) PBDB-T: $P_{H}$

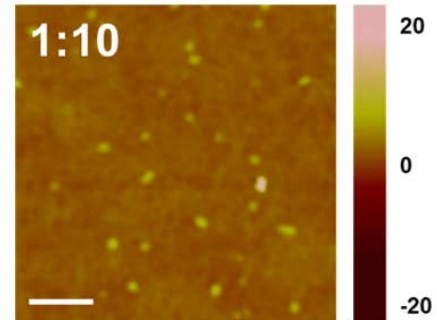

$R_{q}=1.1 \mathrm{~nm}$ (nm)

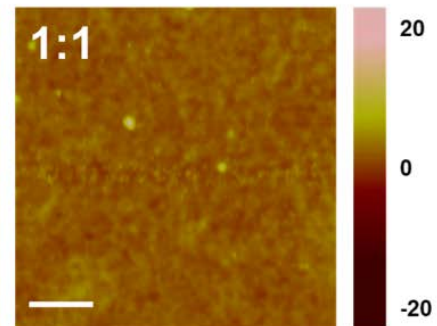

$R_{q}=1.1 \mathrm{~nm}$ (nm)

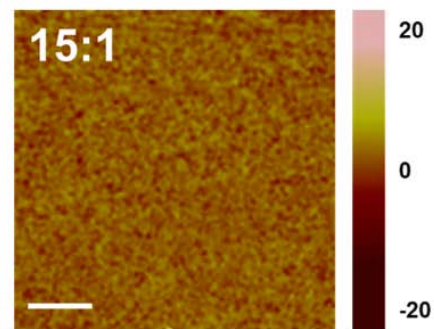

$R_{q}=1.8 \mathrm{~nm}$

Figure S10. AFM height images of BHJ film surfaces before DCB test in (a) PBDB-T: $\mathrm{P}_{\mathrm{L}}$, (b) PBDB-T: $\mathrm{P}_{M}$ and (c) PBDB-T: $\mathrm{P}_{\mathrm{H}}$ blends at different D:A ratios. Scale bars are $1 \mu \mathrm{m}$.
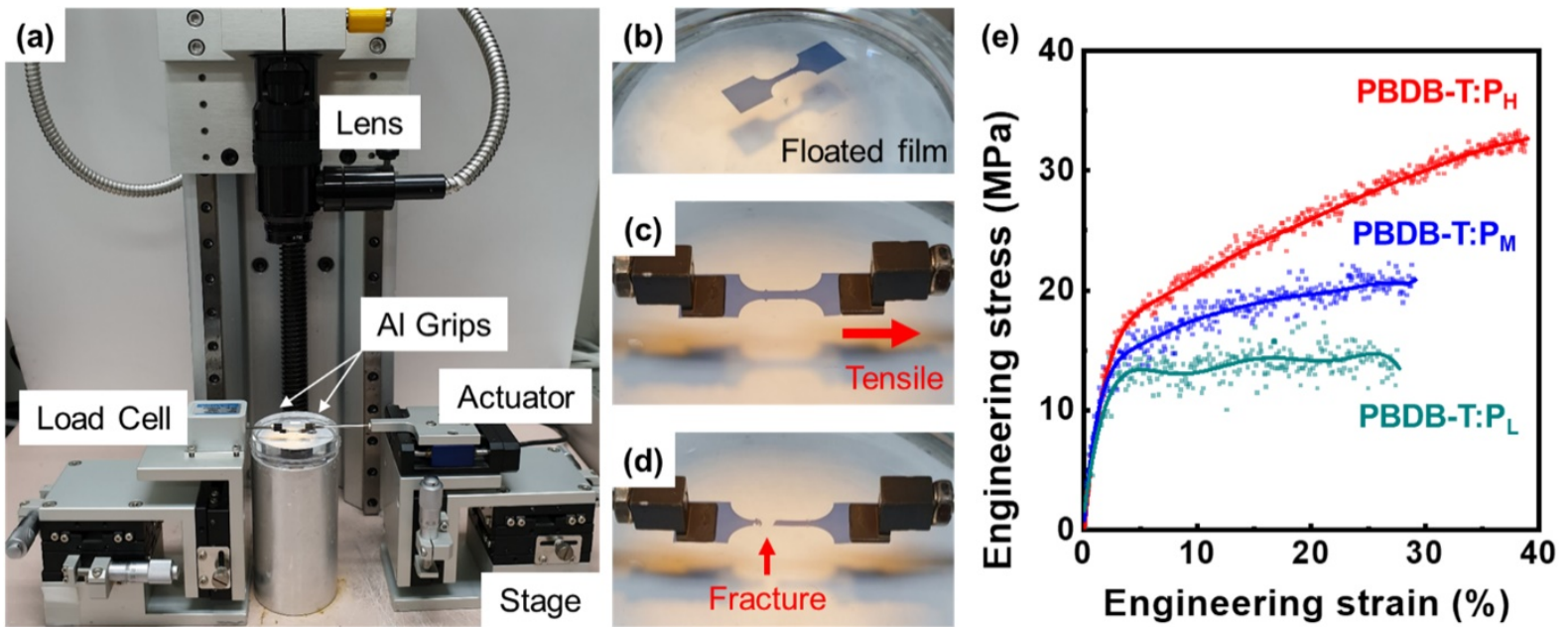

Figure S11. (a) Schematics of pseudo free-standing tensile test setup. (b) Dog-bone shaped BHJ film floated on the water surface, (c) BHJ film fixed to the PDMS-coated aluminum grips, and (d) fractured BHJ film after the tensile test. (e) Stress-strain curve with D:A ratio of 1:1. 